\title{
Quantification of dietary biomarkers in spot urine samples reflects the intake of foods of UK high public health importance
}

\author{
A.J. Lloyd ${ }^{1}$, H. Zubair ${ }^{1}$, N.D. Willis ${ }^{2}$, T. Wilson $^{1}$, L. Xie ${ }^{2}$, K. Tailliart ${ }^{1}$, E.S. Chambers ${ }^{3}$, \\ I. Garcia-Perez ${ }^{3}$, E. Holmes ${ }^{4}$, G. Frost ${ }^{3}$, J.C. Mathers ${ }^{2}$, M. Beckmann ${ }^{1}$ and J. Draper ${ }^{1}$ \\ ${ }^{1}$ Institute of Biological, Environmental and Rural Sciences, Aberystwyth University, Aberystwyth UK, ${ }^{2}$ Human \\ Nutrition Research Centre, Institute of Cellular Medicine, Newcastle University, Newcastle-upon-Tyne, NE4 5PL, \\ ${ }^{3}$ Nutrition and Dietetic Research Group, Division of Diabetes, Endocrinology and Metabolism, Department of \\ Medicine, Hammersmith Hospital Campus, Imperial College London, UK and ${ }^{4}$ Computational and Systems Medicine, \\ Department of Surgery and Cancer, Imperial College London, SW7 2AZ
}

An understanding of causal relations between diet and health is hindered by the lack of robust biological markers of food exposure ${ }^{(1)}$. The rapid development of metabolomics technology offers opportunity for the identification of urine biomarkers for the intake of a

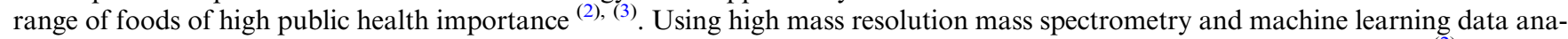
lysis, we have discovered potential urinary biomarkers in controlled clinical studies with a range of analytical techniques ${ }^{(2)}$. To have utility for population monitoring, we aim to validate biomarker performance in free-living individuals using urine samples collected in the home with a minimal impact on normal daily activities.

Two complementary multiple reaction monitoring (MRM) routines using triple quadrupole mass spectrometry (QQQ-MS) have been developed to quantify concurrently dietary exposure biomarkers of more than 20 foods of high public health importance in the UK. MRM quantification of metabolite levels in spot urines collected either before bed time or a first morning void identified a sub-set of potential biomarkers that demonstrated robust linkage with reported dietary intake (examples in Table 1). Figure 1 demonstrates the ability of selected biomarkers to report exposure in relation to muscle meat intake from lunch time to bedtime (Beefburger; 106gm, Chicken breast; 130gm; Processed Ham; 40.5 gm) in 6 free-living individuals. Anserine was strongly, and specifically, associated with poultry intake, whilst the urinary outputs of 3-methyl histidine and carnosine reflect striated muscle intake, with levels substantially reduced when meals contain lower quality, and processed, meats with reduced levels of striated muscle content.

Table 1 .

\begin{tabular}{ll}
\hline Biomarker & Dietary Component \\
\hline$L$-Anserine & Muscle meat \\
Carnosine & Muscle meat \\
3-methylhistidine & Muscle meat \\
Tartarate & Grapes \\
DHPPA-3-sulfate & Wholegrains \\
$D, L$-Sulforaphane- $N$-acetyl- $L$-cysteine & Cruciferous vegetables \\
Proline betaine & Citrus fruits \\
Hydroxyphenylvalerolactone & Procyanidin-rich foods \\
Hippuric acid & Fruit and vegetables \\
\hline
\end{tabular}

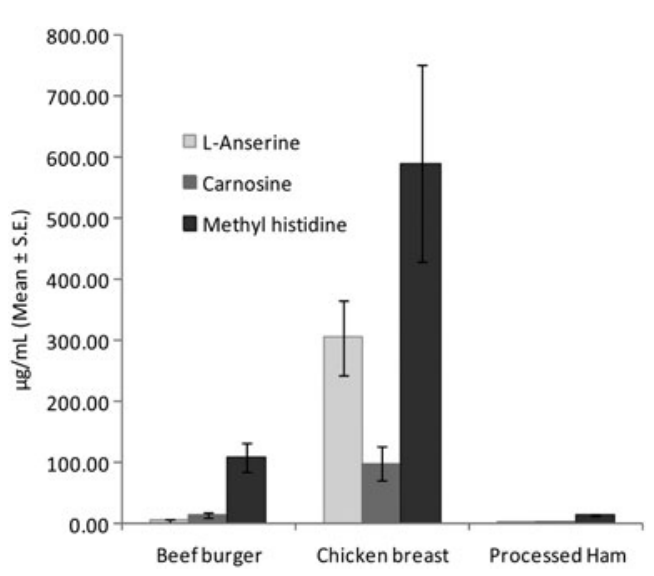

Fig. 1.

The demonstration of the utility of spot urine samples, in combination with robust quantification methods for candidate biomarkers that report multiples components of the UK diet, will allow the future validation of dietary biomarker technology in epidemiological studies.

Funded by MRC grant ref: MR/ J010308/1.

1 Penn et al. (2010) Genes Nutr 5:205-13.

2 Lloyd et al. (2011) Am J Clin Nutr 94:981-91

3 Beckmann M et al. (2013) Proc Nutr Soc. 72:352-61. 